\title{
miR-22-5p revealed as a potential biomarker involved in the acute phase of myocardial infarction via profiling of circulating microRNAs
}

\author{
AGATA MACIEJAK $^{1}$, MAREK KILISZEK ${ }^{2}$, GRZEGORZ OPOLSKI ${ }^{3}$, \\ AGNIESZKA SEGIET $^{4}$, KRZYSZTOF MATLAK $^{5}$, SLAWOMIR DOBRZYCKI $^{6}$, \\ DOROTA TULACZ ${ }^{1}$, GRAZYNA SYGITOWICZ ${ }^{7}$, BEATA BURZYNSKA ${ }^{1}$ and MONIKA GORA ${ }^{1}$
}

\begin{abstract}
${ }^{1}$ Department of Genetics, Institute of Biochemistry and Biophysics, Polish Academy of Sciences, 02-106 Warsaw;
${ }^{2}$ Department of Cardiology and Internal Diseases, Military Institute of Medicine, 04-141 Warsaw; ${ }^{3} 1$ st Chair and

Department of Cardiology; ${ }^{4} 1$ st Faculty of Medicine, Medical University of Warsaw, 02-091 Warsaw;

Departments of ${ }^{5}$ Cardiac Surgery and ${ }^{6}$ Invasive Cardiology, Medical University of Bialystok, 15-089 Bialystok;

${ }^{7}$ Department of Medical Laboratory Diagnostics, Medical University of Warsaw, 02-091 Warsaw, Poland
\end{abstract}

Received April 4, 2016; Accepted July 14, 2016

DOI: $10.3892 / \mathrm{mmr} .2016 .5566$

\begin{abstract}
Acute myocardial infarction (AMI) is a life-threatening episode of coronary artery disease. Recently, circulating myocardial-derived microRNAs (miRNAs) have been reported as potential biomarkers of infarction. The present study aimed to identify differentially expressed miRNAs in patients with ST-segment elevation myocardial infarction that could be potentially dysregulated in response to early myocardial damage. miRNA expression profile analysis was performed using the Serum/Plasma Focus miRNA Polymerase Chain Reaction (PCR) panel of Exiqon A/S (Vedbaek, Denmark) on plasma samples of patients on the first day of AMI (admission) and on samples from the identical patients collected six months following AMI. Selected miRNAs were validated by reverse transcription-quantitative PCR (RT-qPCR) using independent patients with AMI and a control group of patients with a stable coronary artery disease. Thirty-two species of plasma
\end{abstract}

Correspondence to: Dr Beata Burzynska or Dr Monika Gora, Department of Genetics, Institute of Biochemistry and Biophysics, Polish Academy of Sciences, Pawinskiego 5A, 02-106 Warsaw, Poland

E-mail: atka@ibb.waw.pl

E-mail: mgora@ibb.waw.pl

Abbreviations: AMI, acute myocardial infarction; BNP, B-type natriuretic peptide; CAD, coronary artery disease; CI, confidence interval; IPA, ingenuity pathway analysis; miRNA, microRNA; NT-proBNP, N-terminal pro B-type natriuretic peptide; PCA, principal component analysis; RT-qPCR, reverse transcription-quantitative polymerase chain reaction; STEMI, ST-segment elevation myocardial infarction

Key words: circulating microRNAs, microRNA expression profiles, miR-133b, miR-22-5p, acute myocardial infarction
miRNA were differentially expressed $(\mathrm{P}<0.05)$ on admission compared with six months following AMI. Subsequent validation in an independent patient group confirmed that miR-133b and miR-22-5p were significantly up-regulated in the serum of patients with AMI. The receiver operating characteristic (ROC) curve analysis demonstrated a diagnostic utility for miR-22-5p, which has not previously been reported to be associated with AMI. Among the selected miRNAs, miR-22-5p represents a novel promising biomarker for the diagnosis of AMI.

\section{Introduction}

Acute myocardial infarction (AMI) is a life-threatening episode of coronary artery disease, and an as yet unresolved clinical issue with high morbidity and mortality. Insufficient blood supply and oxidative stress result in necrosis of cardiac tissue, pathological remodeling and left ventricular dysfunction (1-3). An early and accurate diagnosis of AMI is essential for an optimal treatment outcome. Therefore, new approaches that are able to complement and improve current strategies for AMI diagnosis are urgently needed.

Recent discoveries have revealed the existence of stable cardiomyocyte-enriched microRNAs (miRNAs) circulating in human blood cells or plasma/serum $(4,5)$. miRNAs are small, non-coding RNA molecules, 20-25 nucleotides long, which inhibit gene expression by promoting messenger RNA (mRNA) degradation or inhibiting translation (6-8). It is noteworthy that numerous studies have revealed that some fraction of the circulating miRNAs is secreted from healthy or damaged cells (9). The fact that these miRNAs are able to be detected in peripheral blood and are relatively stable in serum, plasma and other biofluids makes them potentially useful in aiding diagnosis or guiding therapy through rapid and easy tests eliminating the necessity of performing an invasive procedure $(10,11)$.

The present study aimed to compare the miRNA profiles in plasma samples of patients on the first day of AMI (admission) 


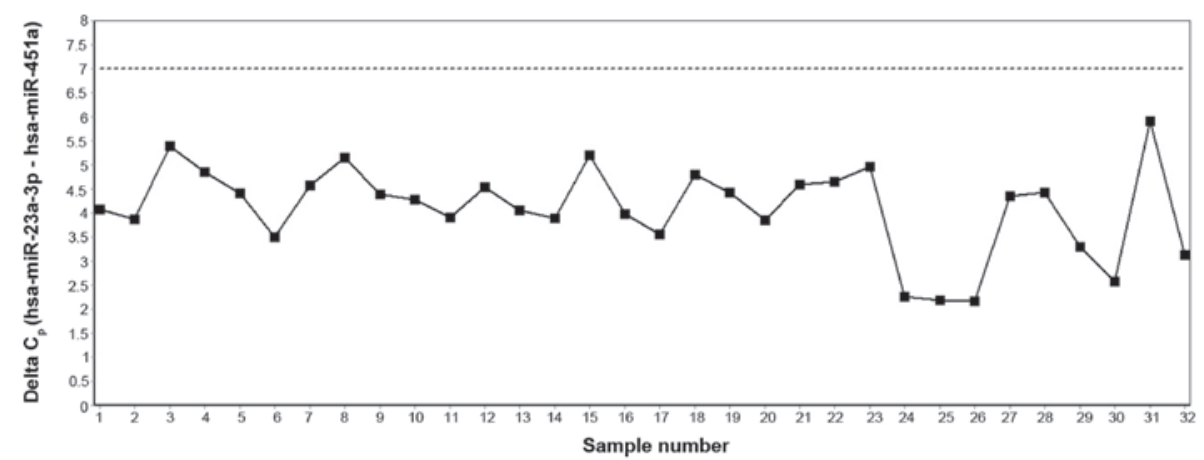

Figure 1. Hemolysis test of plasma samples. miR-451a and miR-23a-3p were determined by RT-qPCR in plasma samples from patients on the first day and six months following MI. The Cp value of miR-23a-3p minus the $\mathrm{Cp}$ value of miR-451a is plotted. The difference does not reach 7 (horizontal dotted line), set as indicative of hemolysis, in any of the samples. RT-qPCR, quantitative reverse transcription-polymerase chain reaction; MI, myocardial infarction.

with those from the identical patients collected six months after AMI (stable phase) in order to identify differentially expressed miRNAs that could be potentially dysregulated in response to early myocardial damage. The most promising miRNAs were additionally studied using a set of AMI serum samples from a second independent cohort and a control group of patients with a stable coronary artery disease (CAD).

\section{Materials and methods}

Patients. Sixteen patients for the study group and fourteen patients for the validation group, diagnosed with ST-segment elevation myocardial infarction (STEMI), were randomly selected from our previously described cohorts of patients admitted to the Medical University of Warsaw and the Medical University of Bialystok (12). The control group comprised seven age- and sex-matched individuals selected from a cohort of patients with a stable CAD and no history of myocardial infarction (MI). The whole cohort of CAD patients has been characterized in our previous study (12).

The design and conduct of this study complied with the Declaration of Helsinki. The protocol of the study was approved by the Ethics Committees of the Medical University of Warsaw and the Medical University of Bialystok. Written informant consent was obtained from all patients.

Plasma and serum collection, and hemolysis assessment. Venous whole blood samples $(4-8 \mathrm{ml})$ were drawn from the patients diagnosed with STEMI at two time points: On the first day of AMI (admission), and six months following AMI using standard phlebotomy techniques. For the study group, plasma was isolated using BD Vacutainer ${ }^{\circledR} \mathrm{CPT}^{\text {TM }}$ glass tubes with sodium citrate (BD Biosciences, Franklin Lakes, NJ, USA), following the manufacturer's protocol. For the validation and control groups, blood samples were drawn into serum separator tubes (Profilab s.c., Warsaw, Poland) according to the manufacturer's instructions. The plasma and serum were transferred into fresh tubes and stored at $-80^{\circ} \mathrm{C}$ prior to subsequent analysis.

Oxyhemoglobin was assayed in all plasma and serum samples analyzed from the study, validation and control groups. Absorbance at $\lambda=414 \mathrm{~nm}$ was measured spectrophotometrically (NanoDrop ND-1000; Thermo Fisher Scientific, Inc., Waltham, MA, USA). Additionally, at the profiling stage, hemolysis in the plasma samples was assessed using two miRNAs available on the Serum/Plasma Focus miRNA Polymerase Chain Reaction (PCR) panel: miRNA-451a, which is specific to erythrocytes, and miRNA-23a-3p, which is unaffected by hemolysis. The $\Delta \mathrm{Cp}$ (crossing point) values for (miR-23a-3p - miR-451a) were calculated. Samples with a $\Delta \mathrm{Cp}$ value $>7.0$ were likely to have undergone hemolysis. Both the absorbance measurements at $\lambda=414 \mathrm{~nm}$ (data not shown) and the $\Delta \mathrm{Cp}$ data (Fig. 1) indicated that no serum and plasma sample was affected by hemolysis.

miRNA isolation, complementary DNA (cDNA) synthesis and quality controls. Total RNA was extracted from $200 \mu \mathrm{l}$ plasma/serum using an miRCURY ${ }^{\mathrm{TM}}$ RNA Isolation kit - Biofluids (Exiqon A/S, Vedbaek, Denmark). To improve the yield and reproducibility between isolations, $1.25 \mu \mathrm{g} / \mathrm{ml} \mathrm{MS2} \mathrm{bacteriophage} \mathrm{RNA} \mathrm{carrier} \mathrm{(Roche}$ Diagnostics GmbH, Mannheim, Germany) was added at the beginning of the procedure. To control isolation efficiency and yield, three synthetic RNA spike-ins (UniSp2, UniSp4 and UniSp5; Exiqon A/S) were added to the samples at concentrations recommended by the manufacturer. Total RNA was eluted with $50 \mu \mathrm{l}$ ribonuclease-free water and stored at $-80^{\circ} \mathrm{C}$ prior to analysis. cDNA was synthesized from purified miRNA using the miRCURY ${ }^{\mathrm{TM}}$ LNA $^{\mathrm{TM}}$ microRNA PCR, Polyadenylation and cDNA synthesis kit II from Exiqon A/S, according to the manufacturer's protocol. During cDNA synthesis, two spike-ins (UniSp6 and cel-miR-39; Exiqon A/S) were added to detect the presence of potential inhibitors in the cDNA synthesis process or in reverse transcription-quantitative PCR (RT-qPCR). The quality control analysis was performed according to the protocol provided in the manual (http://www.exiqon. com/ls/Documents/Scientific/QC-PCR-Panel-Manual.pdf). All samples passed the criteria and were included in further studies.

miRNA profiling. For initial screening, quantification of the miRNA levels in samples taken from patients with AMI [both on the first day of AMI (admission) and six months following AMI] was performed by using the Serum/Plasma Focus microRNA PCR panel, Version V3 (Exiqon A/S) in a 96-well format, which was designed to detect the 179 most expressed miRNAs in human serum/plasma. RT-qPCR reactions were performed using ExiLENT SYBR ${ }^{\circledR}$ Green master mix (Exiqon A/S) according to the protocol provided by the manufacturer. Negative controls (no template) were performed 
Table I. Clinical characteristics of patients from study and validation groups.

\begin{tabular}{|c|c|c|c|}
\hline Characteristic & Study group $(\mathrm{n}=16)$ & Validation group $(n=14)$ & P-value \\
\hline Gender (female/male) & $3 / 13(18.8 \% / 81.2 \%)$ & $0 / 14(0.0 \% / 100.0 \%)$ & 0.200 \\
\hline Age (years) & $54.9 \pm 11.3$ & $58.2 \pm 11.1$ & 0.422 \\
\hline BMI $\left(\mathrm{kg} / \mathrm{m}^{2}\right)$ & $26.8 \pm 2.3$ & $28.0 \pm 4.3$ & 0.345 \\
\hline Smoking & $7(43.8 \%)$ & $8(57.1 \%)$ & 0.715 \\
\hline Hypertension & $4(25.0 \%)$ & $10(71.4 \%)$ & 0.026 \\
\hline Diabetes & $2(12.5 \%)$ & $2(14.3 \%)$ & $>0.999$ \\
\hline Hypercholesterolemia & $9(56.2 \%)$ & $10(71.4 \%)$ & 0.466 \\
\hline Previous MI & $0(0.0 \%)$ & $0(0.0 \%)$ & NA \\
\hline Anterior MI & $9(60.0 \%)$ & $5(35.7 \%)$ & 0.272 \\
\hline Previous revascularization & $0(0.0 \%)$ & $0(0.0 \%)$ & NA \\
\hline Non-coronary atherosclerosis & $0(0.0 \%)$ & $1(7.1 \%)$ & 0.467 \\
\hline $\mathrm{WBC}\left(\mathrm{x} 10^{3} / \mu \mathrm{l}\right)$ & $12.2 \pm 3.3$ & $13.0 \pm 3.6$ & 0.506 \\
\hline NT-proBNP (pg/ml) & $1,052.4(458.1-1,504.3)$ & $784.1(514.5-1,640.0)$ & 0.861 \\
\hline $\operatorname{LVEF}(\%)$ & $49.9 \pm 11.3$ & $41.0 \pm 10.0$ & 0.039 \\
\hline \multicolumn{4}{|l|}{ Medication } \\
\hline Aspirin & $16(100.0 \%)$ & $14(100.0 \%)$ & NA \\
\hline Clopidogrel & $15(93.8 \%)$ & $14(100.0 \%)$ & $>0.999$ \\
\hline Beta blockers & $16(100.0 \%)$ & $13(92.9 \%)$ & 0.467 \\
\hline ACE inhibitors & $16(100.0 \%)$ & $14(100.0 \%)$ & NA \\
\hline Statins & $16(100.0 \%)$ & $14(100.0 \%)$ & NA \\
\hline Diuretics & $6(37.5 \%)$ & $6(42.9 \%)$ & $>0.999$ \\
\hline
\end{tabular}

Data at admission (on the first day of AMI) are presented as the mean value \pm standard deviation or median (first quartile - third quartile) for continuous variables, and the number or percentage of patients for categorical variables. $\mathrm{P}<0.05$ was considered to indicate a statistically significant value. ACE, angiotensin-converting enzyme; BMI, body mass index; LVEF, left ventricular ejection fraction; (A)MI, (acute) myocardial infarction; NA, not applicable; NT-proBNP, N-terminal pro-B-type natriuretic peptide; WBC, white blood cells.

and profiled in an identical manner as for the samples. The amplification was performed in a LightCycler ${ }^{\circledR} 480$ Real-Time PCR system (Roche Diagnostics, Basel, Switzerland). The amplification curves were analyzed using the Roche LC software (version 1.5), both for determination of the $\mathrm{Cp}$ values (by the second derivative method) and for melting curve analysis.

Quantification of individual miRNAs. Selected miRNAs whose levels were found to differ between patients on admission and six months following AMI were subjected to a subsequent validation step by RT-qPCR. The specific microRNA LNA ${ }^{\mathrm{TM}}$ PCR primer sets and ExiLENT SYBR ${ }^{\circledR}$ Green Master Mix (Exiqon $\mathrm{A} / \mathrm{S}$ ) were used to assess the presence of individual miRNAs in serum samples according to the manufacturer's protocol.

miRNA RT-qPCR data analysis. RT-qPCR results were analyzed using the GenEx software, version 6.0 (MultiD Analyses AB, Göthenburg, Sweden). Data obtained for the negative control plate were subtracted from the data for the miRNA PCR panels. Only miRNA species with a Cp value $<37$ and at least 5 points below the negative control $\mathrm{Cp}$ value were included in the data analysis. For the profiling study, the expression data were normalized to a global mean. A logarithmic transformation $\left(\log _{2}\right)$ was used to normalize the expression data in the profiling stage. The geNorm and NormFinder algorithms (Exiqon A/S software) were used to select the reference gene for the validation studies. The data were normalized to miR-19b-3p as a stable endogenous reference gene, and UniSp2 as a stable exogenous reference gene.

Prediction and functional analysis of miRNA targets. Ingenuity Pathway Analysis (IPA; www.ingenuity.com; Qiagen, Inc., Valencia, CA, USA) was used to search mRNA targets for dysregulated miRNAs. To avoid exceeding the maximum gene list size allowed by IPA, the miRNAs were analyzed using the microRNA Target Filter limited to experimentally validated miRNA-mRNA interactions. Target genes were further analyzed for over-represented biological functions and canonical pathways using the IPA database.

Statistical analysis. Statistical analyses were performed using R 3.1.2 software (The R Project for Statistical Computing; https://www.R-project.org). The Shapiro-Wilk test was used to test for normal distribution of continuous variables, and subsequently, continuous variables were expressed as the mean \pm standard deviation for normally distributed ones and the median (first quartile - third quartile) for the variables that deviated from a normal distribution. Categorical variables were presented as frequencies and percentages. Student's t-test (for normally distributed variables) and the Mann-Whitney test (for the variables deviating from a normal distribution) were used to compare continuous variables. Fisher's exact test 
Table II. Differential miRNAs between the first day of AMI and the stable phase following myocardial infarction in the study group.

\begin{tabular}{lcc}
\hline miRNA & Fold change & P-value \\
\hline hsa-miR-133b & 45.764 & $9.0 \mathrm{E}-06$ \\
hsa-miR-133a & 30.127 & $1.6 \mathrm{E}-04$ \\
hsa-miR-208a & 27.074 & $3.0 \mathrm{E}-06$ \\
hsa-miR-1 & 12.139 & $2.0 \mathrm{E}-03$ \\
hsa-miR-30a-5p & 3.643 & $7.0 \mathrm{E}-03$ \\
hsa-miR-629-5p & 2.573 & $4.5 \mathrm{E}-02$ \\
hsa-miR-20b-5p & 2.570 & $2.4 \mathrm{E}-02$ \\
hsa-miR-22-5p & 2.360 & $1.1 \mathrm{E}-02$ \\
hsa-miR-145-5p & 1.776 & $9.0 \mathrm{E}-03$ \\
hsa-miR-22-3p & 1.507 & $2.0 \mathrm{E}-02$ \\
hsa-miR-486-5p & 1.402 & $3.8 \mathrm{E}-02$ \\
hsa-miR-451a & 1.349 & $3.3 \mathrm{E}-02$ \\
hsa-miR-92a-3p & 1.327 & $1.0 \mathrm{E}-02$ \\
hsa-miR-93-5p & 1.133 & $3.1 \mathrm{E}-02$ \\
hsa-let-7i-5p & -1.198 & $1.8 \mathrm{E}-02$ \\
hsa-miR-148b-3p & -1.226 & $3.4 \mathrm{E}-02$ \\
hsa-miR-103a-3p & -1.237 & $1.3 \mathrm{E}-02$ \\
hsa-miR-223-3p & -1.237 & $1.7 \mathrm{E}-02$ \\
hsa-miR-652-3p & -1.251 & $3.6 \mathrm{E}-02$ \\
hsa-miR-26b-5p & -1.257 & $4.2 \mathrm{E}-02$ \\
hsa-miR-107 & -1.272 & $7.0 \mathrm{E}-03$ \\
hsa-miR-199a-3p & -1.278 & $3.0 \mathrm{E}-02$ \\
hsa-miR-151a-5p & -1.326 & $9.0 \mathrm{E}-03$ \\
hsa-miR-30b-5p & -1.414 & $3.0 \mathrm{E}-03$ \\
hsa-miR-181a-5p & -1.436 & $4.6 \mathrm{E}-02$ \\
hsa-miR-142-3p & -1.693 & $3.6 \mathrm{E}-04$ \\
hsa-miR-374b-5p & -1.829 & $3.6 \mathrm{E}-04$ \\
hsa-miR-335-5p & -2.384 & $1.5 \mathrm{E}-02$ \\
hsa-miR-505-3p & -2.873 & $5.0 \mathrm{E}-03$ \\
hsa-miR-885-5p & -2.898 & $4.0 \mathrm{E}-02$ \\
hsa-miR-326 & -3.535 & $7.0 \mathrm{E}-03$ \\
hsa-miR-301a-3p & -4.147 & $7.0 \mathrm{E}-03$ \\
\hline & & \\
\hline
\end{tabular}

Data are presented as the fold change relative to the expression of patients six months following AMI. P-values were calculated using a paired two-tailed Student's t-test. Only miRNA species with $\mathrm{P}<0.05$ are shown. AMI, acute myocardial infarction; miRNA, microRNA.

was used to compare categorical variables. Statistical significance between miRNAs that were differentially expressed in study and validation groups was determined using either a paired, two-tailed Student's t-test (admission compared with six months following AMI) or an unpaired, two-tailed Student's t-test (admission compared with the control group). $\mathrm{P}<0.05$ was taken to indicate a statistically significant value. Principal component analysis (PCA) was performed using GenEx software (version 6.0; MultiD Analyses AB). Receiver operating characteristic (ROC) curve analysis and the area under the curve (AUC) were used to estimate the ability of biomarkers to distinguish the AMI group from the control

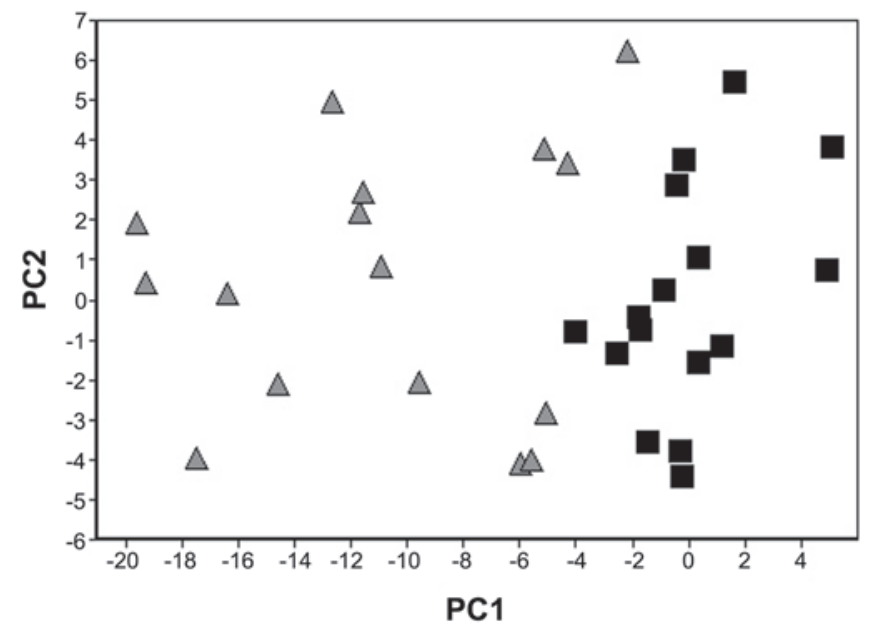

Figure 2. Principal component analysis of plasma miRNA profiles. Principal component analysis of the miRNA expression data clusters plasma collected on admission (grey triangles) and six months following acute myocardial infarction (black squares) into their respective groups. Normalized values were used for principal component analysis. miRNA, microRNA.

group. The optimal cut-off points for each miRNA were determined using the highest sum of sensitivity and specificity.

\section{Results}

Patient characteristics. In the present study, patients with STEMI who were treated with primary percutaneous revascularization were included. The mean age of participants was $54.9 \pm 11.3$ years for the study group $(n=16)$ and $58.2 \pm 11.1$ years for the validation group $(n=14)$. Clinical characteristics of patients from the two groups are shown in Table I.

Identification of differentially expressed miRNAs in the plasma of patients with AMI. miRNA profiling was performed on plasma samples derived from patients on the first day of AMI $(\mathrm{n}=16)$, and on samples from the identical patients collected six months following AMI ( $\mathrm{n}=16$, stable phase), which reduced the impact of inter-individual variability. Following data analysis, miRNA candidates were selected on the basis of fulfillment of the criterion of significance $(\mathrm{P}<0.05)$ in the comparison between admission and six months following AMI. A total of 32 miRNAs (14 up- and 18 down-regulated) were differentially quantified in the acute phase of MI compared with the stable phase following MI (Table II).

PCA was performed on the miRNA results from the analyzed samples to determine how the 32 differentially expressed miRNAs were distributed among the samples from the first day of AMI, and those collected six months afterwards. As shown in Fig. 2, the PCA clearly separated the plasma samples on admission from those collected six months following AMI. This suggests that the observed miRNA differences are associated with the pathophysiology of MI, and these miRNAs might constitute an early biomarker signature for AMI.

Validation of selected miRNAs in an independent group of patients with AMI. miRNA candidates for validation were selected following an extensive review of the literature on the basis of their inferred relevance to cardiovascular 
Table III. RT-qPCR quantification of selected miRNAs on admission vs. six months following AMI in study and validation groups.

Admission vs. six months

\begin{tabular}{lcccr}
\cline { 2 - 3 } miRNA & \multicolumn{2}{c}{ Study group } & \multicolumn{2}{c}{ Validation group } \\
\cline { 2 - 4 } & Fold change & P-value & & Fold change \\
\hline hsa-miR-133b & 45.764 & $<0.001$ & 5.449 & $<0.05$ \\
hsa-miR-22-5p & 2.360 & $<0.05$ & 4.872 & $<0.01$ \\
hsa-miR-374b-5p & -1.829 & $<0.001$ & 1.642 & NS \\
\hline
\end{tabular}

Results were normalized to miR-19b-3p and UniSp2. Data for the study group are taken from Table II. miRNA, microRNA; NS, non-significant; RT-qPCR, reverse transcription-quantitative polymerase chain reaction.

Table IV. RT-qPCR quantification of the two selected miRNAs on admission vs. the control group in the validation group.

\begin{tabular}{lcc}
\hline & \multicolumn{2}{c}{ Admission vs. control group } \\
\cline { 2 - 3 } & \multicolumn{2}{c}{ Validation group } \\
\cline { 2 - 3 } miRNA & Fold change & P-value \\
\hline hsa-miR-133b & 7.273 & $<0.05$ \\
hsa-miR-22-5p & 4.505 & $<0.01$ \\
\hline
\end{tabular}

Results were normalized to miR-19b-3p and UniSp2. miRNA, microRNA; RT-qPCR, reverse transcription-quantitative polymerase chain reaction.

disease. Additionally, the potential candidates were filtered for highly expressed miRNAs according to inspection of their raw $\mathrm{Cp}$ values in individual samples. The validation was performed on serum samples of an independent patient group on admission $(n=14)$ and samples from the identical patients collected six months following AMI $(n=14)$, and a control group $(n=7)$. The levels of these miRNAs were quantified using RT-qPCR for individual miRNAs. Three miRNAs were further investigated: miR-133b, which is known to be associated with MI; miR-374b-5p, which limited literature has suggested has an involvement in MI; and miR-22-5p, which has not yet been reported to be associated with MI, and therefore may be a possible novel biomarker. Two of the miRNAs, miR-133b and miR-22-5p, demonstrated significant differences in the comparison between admission and six months following AMI, the direction and magnitude of the changes reflecting reasonably well those found in the profiling stage (Table III). These two miRNAs were further investigated in the patients with AMI and the control group. As shown in Table IV, the expression levels of miR-133b and miR-22-5p were significantly increased in patients with AMI compared with the control group.

Diagnostic accuracy of selected circulating miRNAs. To evaluate the diagnostic value of miR-133b and miR-22-5p as potential biomarkers of AMI, ROC curve analysis, together
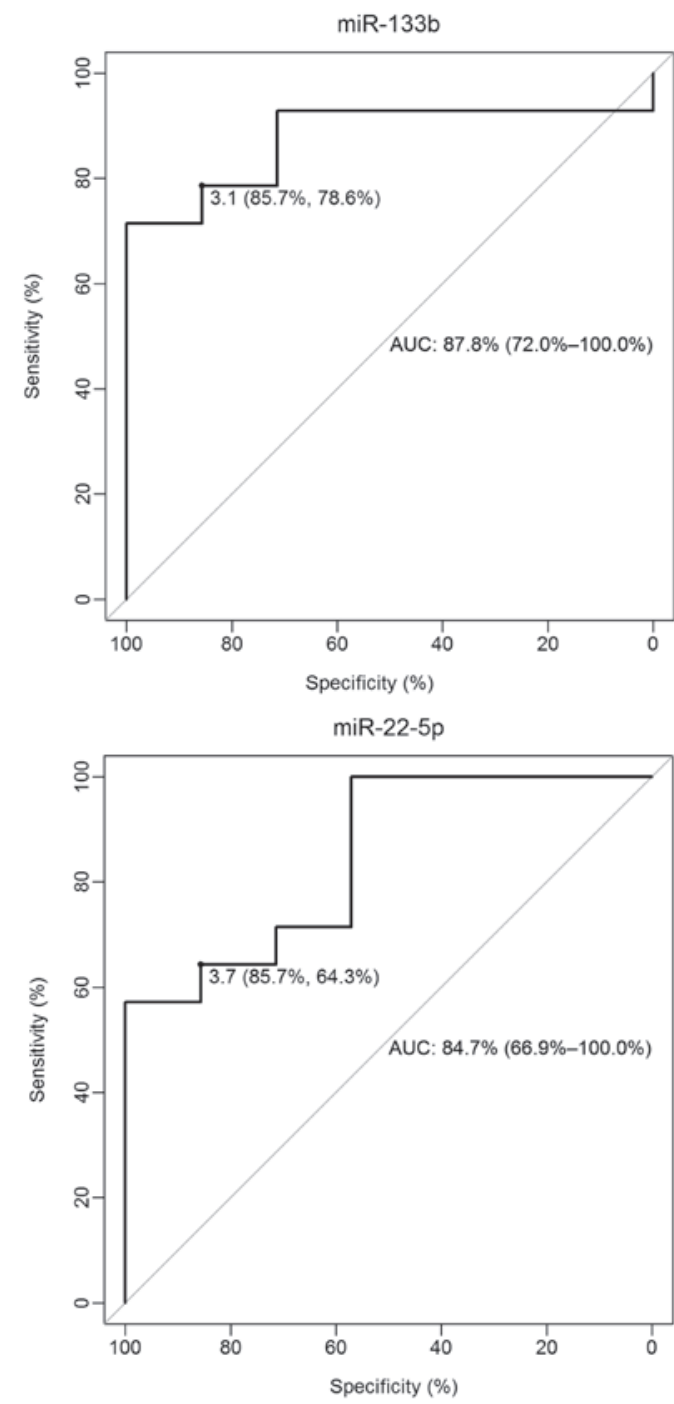

Figure 3. ROC curves for miR-133b and miR-22-5p. AUC, area under the curve; ROC, receiver operating characteristic.

with calculation of the AUC, was performed. As shown in Fig. 3, the ROC curves of miR-133b and miR-22-5p reflected a good separation between the patients with AMI and the control group, with AUC measurements of 87.8\% [95\% confidence 
Cellular Development

Cell Morphology

Cellular Growth and Proliferation

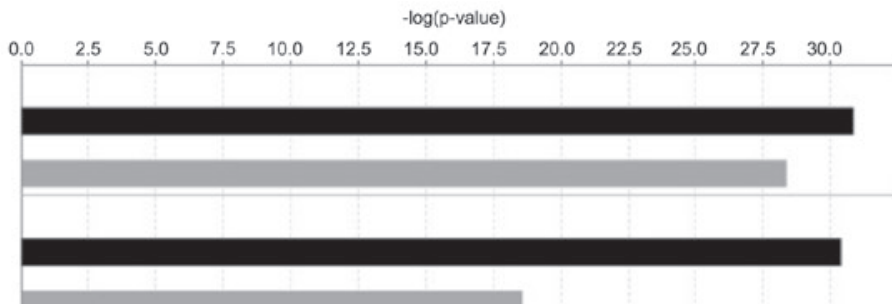

Cellular Movement

Cell Death and Survival

Cell Cycle

DNA Replication, Recombination, and Repair

Gene Expression

Cellular Assembly and Organization

Cellular Function and Maintenance

Molecular Transport

Cell-To-Cell Signaling and Interaction

Post-Translational Modification

Protein Synthesis

Nucleic Acid Metabolism

Small Molecule Biochemistry

Cell Signaling
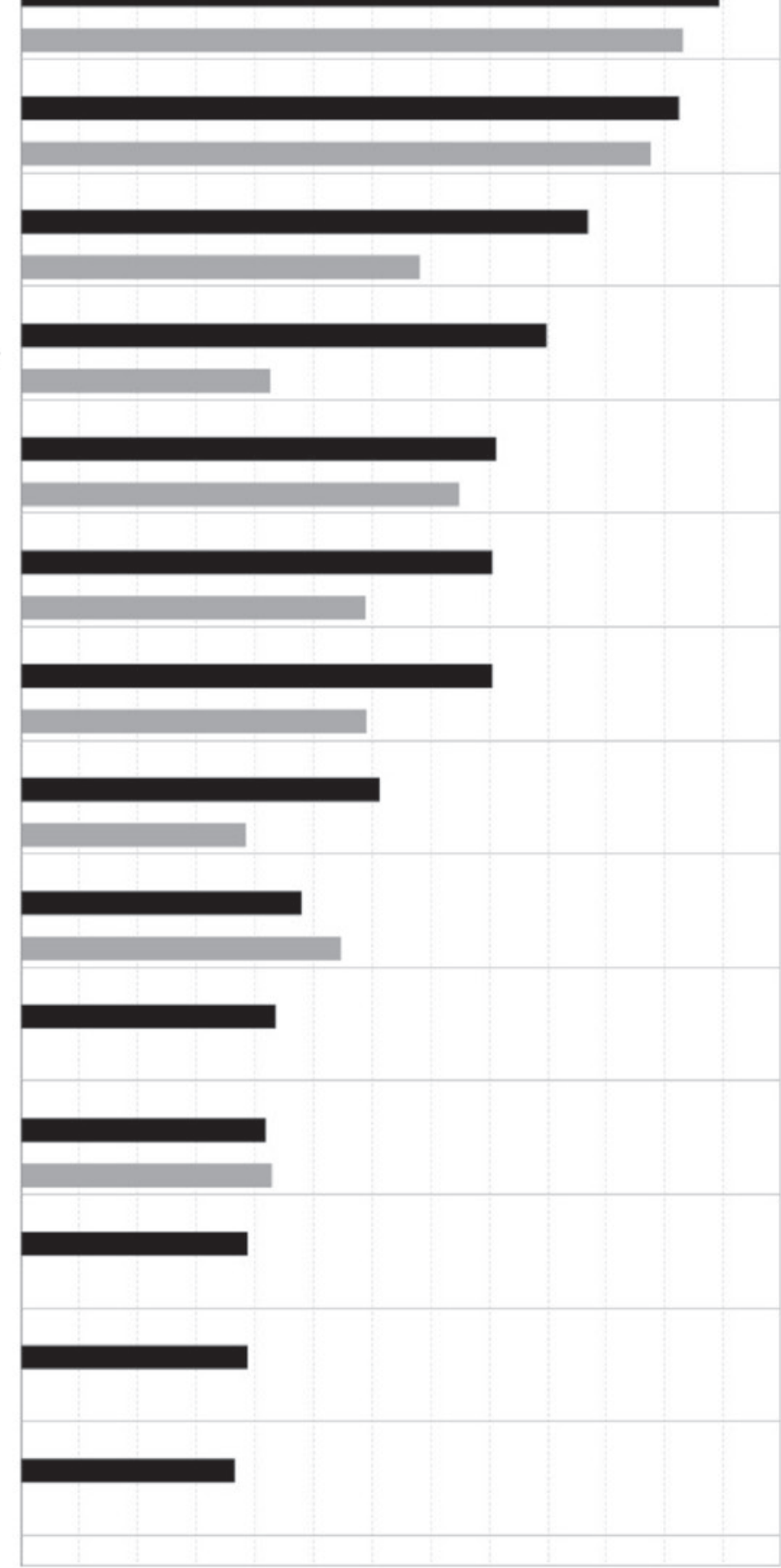

Figure 4. Molecular and cellular functions associated with differentially expressed miRNAs. Ingenuity pathway analysis of miRNA sets differentially expressed on admission compared with six months after acute myocardial infarction was performed. The x-axis of the diagram displays the significance, which was calculated using the Fisher exact test and expressed as the negative exponent of the P-value. Pathways associated with up-regulated miRNAs are shown in black, whereas those pathways associated with down-regulated miRNAs are shown in grey. miRNA, microRNA. 
Cardiac Hypertrophy Signaling

Inhibition of Angiogenesis by TSP1

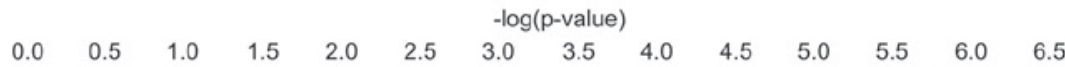

Hypoxia Signaling in the Cardiovascular System

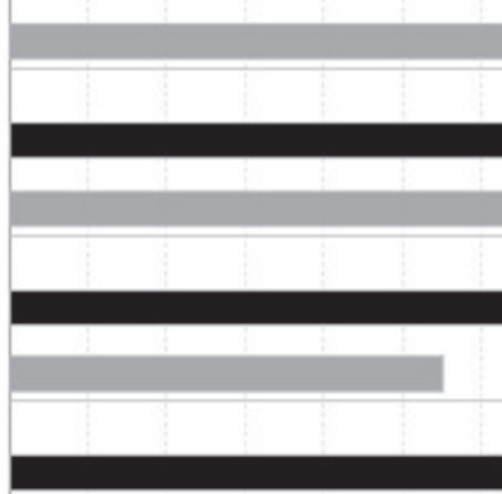

Role of NFAT in Cardiac Hypertrophy

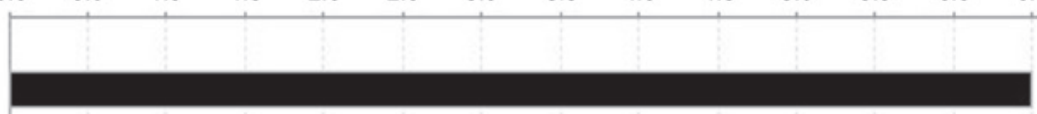

HIF1 $\alpha$ Signaling

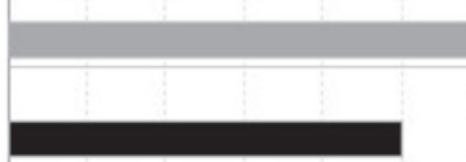

Factors Promoting Cardiogenesis in Vertebrates

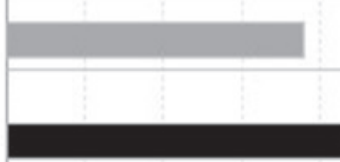

Factors Promoting Cardiogenesis in Vertebrates

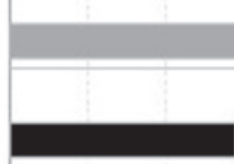

Angiopoietin Signaling

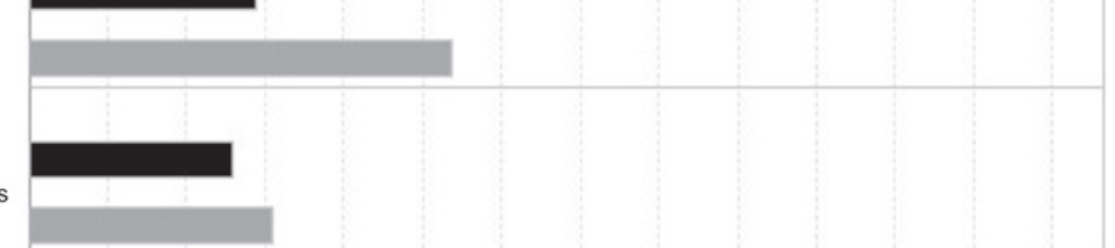

Cardiomyocyte Differentiation via BMP Receptors

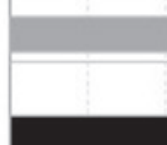

P2Y Purigenic Receptor Signaling Pathway

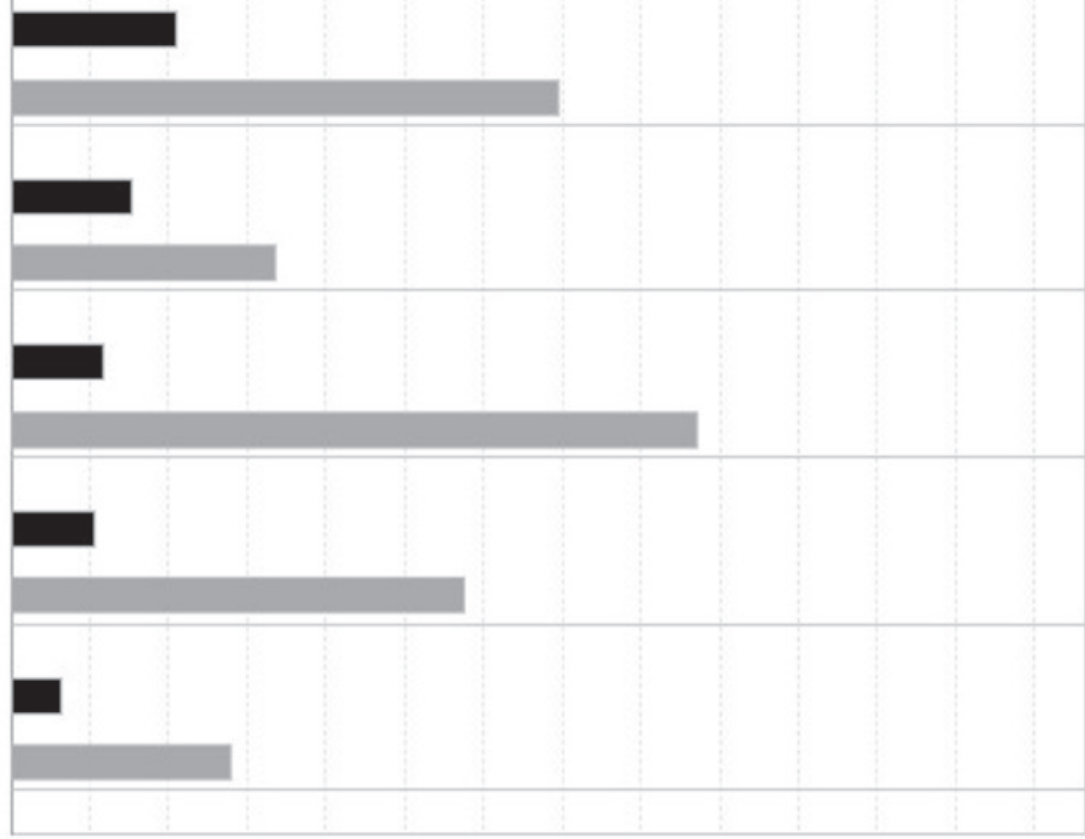

Endothelin-1 Signaling

Thrombin Signaling

Cardiac $\beta$-adrenergic Signaling

Figure 5. Predicted cardiovascular signaling pathways regulated by miRNAs differentially expressed in AMI. Ingenuity pathway analysis of miRNA sets differentially expressed on admission compared with six months following AMI was performed. The $\mathrm{x}$-axis of the diagram displays the significance, which was calculated using the Fisher exact test and expressed as the negative exponent of the P-value. Pathways associated with up-regulated miRNAs are shown in black, whereas those pathways associated with down-regulated miRNAs are shown in grey. AMI, acute myocardial infarction; miRNA, microRNA; TSP-1, thrombospondin 1; NFAT, nuclear factor of activated T-cells; HIF-1 $\alpha$, hypoxia-inducible factor $1 \alpha$; BMP, bone morphogenetic protein. 
interval (CI): 72.0-100.0] and 84.7\% (95\% CI: 66.9-100.0), respectively. ROC curves yielded an optimal cut-off value of 3.1 for miR-133b, with a sensitivity of $78.6 \%$ and a specificity of $85.7 \%$, and an optimal cut-off value of 3.7 for miR-22-5p, with a sensitivity of $64.3 \%$ and a specificity of $85.7 \%$. These results suggest that miR-133b and miR-22-5p are of good diagnostic value for patients with AMI.

\section{Discussion}

Previous studies have revealed that heart-specific miRNAs are released into the circulation during AMI, and therefore may be used to detect and monitor myocardial injury $(13,14)$. In the present study it has been confirmed that the well-known 'cardiac miRNAs' of ongoing early myocardial damage, miR-1, miR-133a, miR-133b and miR-208a, are significantly up-regulated in AMI. An additional 28 differentially expressed miRNAs that were apparently associated with AMI were also identified. To determine the biological significance of miRNAs dysregulated in AMI, in silico target prediction was performed using the IPA software (Qiagen, Inc.). Targeting information was available for 22 out of the 32 miRNAs in the database, resulting in a total of 412 experimentally validated target mRNAs for up-regulated miRNAs, and a total of 304 experimentally validated mRNAs for down-regulated miRNAs. Functional analysis revealed that targets for both up- and down-regulated miRNAs were generally involved in identical molecular and cellular functions (Fig. 4). Only post-translational modification, nucleic acid metabolism, small molecule biochemistry and cell signaling were predicted to be associated with up-regulated miRNAs. In addition, several pathways involved in cardiovascular signaling were revealed to be associated with the canonical pathway analysis, the most important being cardiac hypertrophy signaling, inhibition of angiogenesis by thrombospondin 1 and hypoxia signaling in the cardiovascular system (Fig. 5). These findings reveal that the identified miRNAs could have a role in the pathogenesis of MI through their ability to negatively regulate the expression of genes that govern processes important for myocardial function.

Numerous biochemical biomarkers of MI are commonly used in clinical practice [e.g. cardiac troponins ( $\mathrm{Tn}$ ) I and T, creatinine kinase isoenzyme $\mathrm{MB}, \mathrm{N}$-terminal pro B-type natriuretic peptide and B-type natriuretic peptide] (15). However, it should be noted that an increase in the levels of these biochemical biomarkers above reference values may also occur in various other disease states not necessarily associated with MI (16). Therefore, selected miRNAs or miRNA sets, particularly when combined with clinical parameters, are likely to be more specific biomarkers of MI. Additionally, studying their mechanism of action should provide an improved understanding of the changes that occur in the myocardium, and determine the potential role of extracellular miRNAs as paracrine signaling molecules.

The up-regulation of miR-133b and miR-22-5p in two independent patient groups using serum or plasma confirmed the high diagnostic value of these miRNAs. The ROC curve analysis revealed that the AUCs of miR-133b and miR-22-5p were 87.8 and $84.7 \%$, indicating that they may be clinically practicable biomarkers for AMI diagnosis. The major novel finding reported in the present study is the up-regulation of miR-22-5p in the acute phase of STEMI. To date, mir-22-3p originating from the same hairpin has been studied in depth to elucidate its role in cardiovascular remodeling (17) and heart failure (18). To the best of our knowledge, no previous data regarding a role for miR-22-5p in cardiovascular diseases is available, albeit a recent study has demonstrated that up-regulation of mmu-miR-22-5p may prevent myocardium regeneration in 7-day-old mice (19).

In conclusion, the present study has reported an altered miRNA expression profile associated with AMI. A group of 32 circulating miRNAs that are significantly up- or down-regulated in AMI compared with the stable phase of the disease has been described. The circulating miRNA, miR-22-5p, has been identified as a novel diagnostic biomarker of AMI.

\section{Acknowledgements}

We would like to thank the patients for their participation in this study. We thank Katarzyna Rawa for technical assistance in the miRNA profiling analysis. This work was supported by the National Science Centre, Poland (grant no. 2014/13/N/NZ5/01403) and The National Centre for Research and Development, Poland (grant no. N R13 0001 06).

\section{References}

1. Hori $\mathrm{M}$ and Nishida K: Oxidative stress and left ventricular remodeling after myocardial infarction. Cardiovase Res 81: 457-464, 2009.

2. Sutton MG and Sharpe N: Left ventricular remodeling after myocardial infarction: Pathophysiology and therapy. Circulation 101: 2981-2988, 2000.

3. White HD and Chew DP: Acute myocardial infarction. Lancet 372: 570-584, 2008.

4. Gilad S, Meiri E, Yogev Y, Benjamin S, Lebanony D, Yerushalmi N, Benjamin $\mathrm{H}$, Kushnir M, Cholakh $\mathrm{H}$, Melamed N, et al: Serum microRNAs are promising novel biomarkers. PLoS One 3: e3148, 2008.

5. Chen X, Ba Y, Ma L, Cai X, Yin Y, Wang K, Guo J, Zhang Y, Chen J, Guo X, et al: Characterization of microRNAs in serum: A novel class of biomarkers for diagnosis of cancer and other diseases. Cell Res 18: 997-1006, 2008.

6. Bartel DP: microRNAs: Genomics, biogenesis, mechanism, and function. Cell 116: 281-297, 2004.

7. Ambros V: The functions of animal microRNAs. Nature 431: 350-355, 2004.

8. Inui M, Martello G and Piccolo S: MicroRNA control of signal transduction. Nat Rev Mol Cell Biol 11: 252-263, 2010.

9. Xu J, Zhao J, Evan G, Xiao C, Cheng Y and Xiao J: Circulating microRNAs: Novel biomarkers for cardiovascular diseases. J Mol Med (Berl) 90: 865-875, 2012.

10. Oliveira-Carvalho V, Carvalho VO, Silva MM, Guimarães GV and Bocch EA: MicroRNAs: A new paradigm in the treatment and diagnosis of heart failure? Arq Bras Cardiol 98: 362-369, 2012 (In English, Portuguese, Spanish).

11. Katoh M: Therapeutics targeting angiogenesis: Genetics and epigenetics, extracellular miRNAs and signaling networks (Review). Int J Mol Med 32: 763-767, 2013.

12. Maciejak A, Kiliszek M, Michalak M, Tulacz D, Opolski G, Matlak K, Dobrzycki S, Segiet A, Gora M and Burzynska B: Gene expression profiling reveals potential prognostic biomarkers associated with the progression of heart failure. Genome Med 7: 26, 2015.

13. Wang GK, Zhu JQ, Zhang JT, Li Q, Li Y, He J, Qin YW and Jing Q: Circulating microRNA: A novel potential biomarker for early diagnosis of acute myocardial infarction in humans. Eur Heart J 31: 659-666, 2010.

14. Białek S, Górko D, Zajkowska A, Kołtowski Ł, Grabowski M, Stachurska A, Kochman J, Sygitowicz G, Małecki M, Opolski G and Sitkiewicz D: Release kinetics of circulating miRNA-208a in the early phase of myocardial infarction. Kardiol Pol 73: 613-619, 2015. 
15. Lindahl B: Acute coronary syndrome-the present and future role of biomarkers. Clin Chem Lab Med 51: 1699-1706, 2013.

16. Iqbal N, Wentworth B, Choudhary R, Landa Ade L, Kipper B, Fard A and Maisel AS: Cardiac biomarkers: New tools for heart failure management. Cardiovasc Diagn Ther 2: 147-164, 2012.

17. Huang ZP and Wang DZ: miR-22 in cardiac remodeling and disease. Trends Cardiovasc Med 24: 267-272, 2014.
18. Goren Y, Kushnir M, Zafrir B, Tabak S, Lewis BS and Amir O: Serum levels of microRNAs in patients with heart failure. Eur J Heart Fail 14: 147-154, 2012.

19. Liu HL, Zhu JG, Liu YQ, Fan ZG, Zhu C and Qian LM: Identification of the microRNA expression profile in the regenerative neonatal mouse heart by deep sequencing. Cell Biochem Biophys 70: 635-642, 2014. 